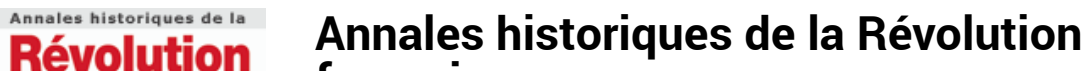

française française

330 | octobre-décembre 2002

Provinces-Paris

\section{Steven L. KAPLAN, La fin des corporations, Paris, Fayard, 2001, $740 \mathrm{p}$.}

Jean-Clément Martin

\section{(2) OpenEdition \\ 12 Journals}

Édition électronique

URL : https://journals.openedition.org/ahrf/3903

DOI : 10.4000/ahrf.3903

ISSN : 1952-403X

Éditeur :

Armand Colin, Société des études robespierristes

Édition imprimée

Date de publication : 1 décembre 2002

Pagination : 186-190

ISSN : 0003-4436

Référence électronique

Jean-Clément Martin, "Steven L. KAPLAN, La fin des corporations, Paris, Fayard, 2001, 740 p. »,

Annales historiques de la Révolution française [En ligne], 330 | octobre-décembre 2002, mis en ligne le 16 avril 2008, consulté le 24 avril 2022. URL : http://journals.openedition.org/ahrf/3903 ; DOI : https:// doi.org/10.4000/ahrf.3903

Ce document a été généré automatiquement le 24 avril 2022.

Tous droits réservés 


\title{
Steven L. KAPLAN, La fin des corporations, Paris, Fayard, 2001, $740 \mathrm{p}$.
}

\author{
Jean-Clément Martin
}

1 Depuis vingt ans Steven Kaplan s'est imposé comme l'un des meilleurs historiens du monde du travail en France à la fin du XVIII ${ }^{e}$ siècle, en même temps qu'il a publié des ouvrages imposants consacrés à la commémoration du bicentenaire de la Révolution et à la boulangerie actuelle. Ce livre est lui aussi d'un volume considérable, 740 pages dont plus d'une centaine de pages de notes qui sont souvent en elles-mêmes des éléments importants dans la démonstration. Il peut apparaître autant comme une somme sur la fin des corporations qu'une proposition nouvelle d'analyse du mouvement sans-culotte, mais aussi comme une réflexion sur l'historiographie française et anglo-saxonne consacrée à la Révolution française, ou encore comme une contribution volontairement impertinente dans le débat contemporain sur le libéralisme et l'étatisme. Avec une alacrité bien connue, soutenue par un style qui recourt parfois à des métaphores ou à des digressions provocantes (comme le titre du chapitre XII : « Encore un mois d'août fatal : la route accidentée vers l'abolition »), l'ouvrage commence par le rappel de ce qui peut être un "paradoxe français ", la dénonciation récurrente de tout ce qui peut passer pour une corporation, alors que les impasses du libéralisme comme de l'étatisme suscitent des interrogations qui conduisent à des réinventions néo-corporatistes, si bien que l'épisode corporatiste de l'État français ne peut pas apparaître comme totalement étranger à l'histoire nationale. Le but avoué de l'entreprise est donc de brosser le cadre de pensée qui a vu la disparition officielle des corporations en France, au printemps 1791, sous l'effet conjoint des lois Allarde et Le Chapelier, sans tomber dans les a priori qui sont ordinairement requis pour les comprendre mais au contraire en exposant la complexité et l'ambiguïté des mouvements qui ont conduit les Français à ce choix. Pour cela, le livre s'attache à retracer l'histoire qui s'est déroulée de 1776 à 1791 - avec des incursions en amont et en aval - de Turgot à Le Chapelier, prenant au passage l'histoire de la Révolution en écharpe ; on en verra les conséquences plus loin. 
2 La trame du livre peut être résumée facilement, puisque la chronologie est respectée et l'auteur a pris le soin de donner en tête de chaque chapitre un résumé, la conclusion fournissant un ultime rappel de l'ensemble. L'histoire commence donc au moment où le ministère Turgot, prolongeant des critiques antérieures, entend supprimer les corporations, les rationaliser et les réorganiser, pour les assujetir administrativement d'un côté et ouvrir le marché du travail à la loi de l'offre et de la demande de l'autre, insistant sur la nécessité de la liberté. La démarche est l'aboutissement d'un double processus, la critique de l'administration royale d'un ensemble peu ordonné et la mise en cause par les Lumières de ce qui apparait comme un anachronisme. Les physiocrates ont gagné, d'autant plus que les concours d'académies ont préparé l'opinion à la décision. La conviction est partagée que le contrôle de l'Etat est inutile, car l'intérêt même du marchand ou du fabricant le pousse à être de bonne foi - position dont on voit bien les résonances politiques ultérieures. La difficulté naît pourtant de la position ambiguë de l'État, qui a taxé jusque-là les corporations et qui garantit leur endettement. Les mesures économiques sont donc inséparables des mesures fiscales et induisent aussi des mesures politiques et même morales. Si la démarche du ministère est logique, parce que nombre d'administrateurs sont acquis aux Lumières, elle est fragile car des oppositions sont tenaces entre interventionnistes et libéraux, entre lesquels des réformateurs tentent de trouver des compromis, et même risquée car cela rappelle des souvenirs fâcheux datant de 1716 ou de 1771, quand l'État avait entrepris de contrôler, voire de rénover, les corporations. Si bien que l'image même de l'État est trouble, un réel libéralisme peut côtoyer le vieux fonds colbertiste d'une administration interventionniste et fiscalement toujours avide. Le camp adverse n'est pas plus unifié, sous le vernis unitaire des corporations se cachent des disparités entre les corps, entre les villes, si bien que des volontés réformatrices ne sont pas absentes, même si les identités profondes ne doivent pas être remises en cause. Enfin, les parlements jouent leur rôle dans cette pièce, défendant l'indépendance des corps contre les empiètements royaux, mais s'affirmant clairement dirigistes pour faire face aux disettes. Une conclusion essentielle à cette étape est que les critiques exprimées dans les années 1770 vis-à-vis des corporations ne sont certes pas inédites, mais qu'elles sont dorénavant portées par l'air du temps d'une part et qu'elles sont soutenues par le roi et par (une partie de) l'administration. Le roi est donc modernisateur, il convient même de parler d'un «libéralisme royal» (p.74) mais qui est dans la nécessité d'imposer ses décisions dans un premier temps. Ainsi chaque catégorie d'acteurs exprime-t-elle des aspirations contradictoires.

3 Dans ce jeu complexe, Turgot introduit en février 1776 des mesures qui suppriment les corporations mais qui renforcent les inégalités économiques, elles sont lues par les tenants des corporations comme destructrices de l'ordre global, tandis que les compagnons les tiennent pour la possibilité d'obtenir une liberté plus grande. Il s'agit d'une véritable inversion des valeurs sociales, d'une «carnavalisation» de la société, justifiant l'insubordination des compagnons, même introduisant un renversement généralisé des valeurs sociales comme le craignent les parlementaires qui défendent les corps intermédiaires comme base indispensable à l'équilibre social. Ces mesures sont pourtant très inégalement appliquées, voire pas du tout dans de nombreuses provinces où les parlements, voire les administrateurs locaux, font une résistance efficace, si bien qu'elles sont abandonnées en mai 1776, ce qui entraîne des bouleversements véritables; car il ne s'agit pas d'un retour à la situation antérieure, mais au contraire de la création d'une situation nouvelle. Le rétablissement des corporations qui n'ont pas 
été supprimées s'accompagne d'une redéfinition générale des corporations, de leurs rapports entre elles comme avec l'État, puisqu'il ne s'agit rien de moins qu'une véritable refondation des corporations pour tenir compte des relations réelles entre les corps de métier, sur fond de reprise en main des ouvriers; Turgot a bien provoqué un «cataclysme». Un des exemples est donné par la place accordée aux femmes dans le nouvel ordre, tandis qu'elle est toujours refusée aux Juifs. En même temps, les ouvriers déçus par le retrait de Turgot persistent dans leur fronde, enfin les critiques contre le libéralisme se font plus vives, dénonçant un danger réel pour la société.

En tout état de cause, l'opération profite inégalement aux maitres, car selon les corps et les provinces, la refondation des corporations s'accomplit inégalement, tenant compte des résistances antérieures, de l'inexistence aussi parfois des corporations en tant que telles. En outre ces refondations introduisent l'agrégation à côté de la maîtrise, créant un nouveau statut flou dans la communauté des maîtres. En résultent une fronde continue des ouvriers et des compagnons, une division parmi les maîtres dans chaque corporation, comme entre les corporations, puisqu'une partie est reconduite tandis que d'autres sont confrontées à des amalgames qu'elles refusent, enfin une crise de recrutement des maitres et la confirmation pour beaucoup qu'il est possible de s'établir hors du contrôle corporatif.

5 Même s'il est difficile de connaître l'état exact des luttes sociales, il est assuré que les années 1780 voient la montée de l'emprise des idées des Lumières, de la volonté réformatrice de l'État, mais aussi des difficultés économiques, le tout entraînant une augmentation des tensions sociales. Le processus n'est ni linéaire, ni simple, mais combiné, le résultat est complexe, mais débouche pour partie sur la montée de l'individualisme, tant patronal qu'ouvrier, sur fond de critiques de la part des corporations reconstituées contre les «faux ouvriers" qui s'installent en toute impunité. Pendant ce temps, le faubourg Saint-Antoine défend bec et ongle sa liberté d'entreprise, hors corporation, et son image d'excellence contre les accusations des chefs des grands corps.

Dans ce climat troublé, la convocation des États généraux introduit un nouvel élément perturbant, puisque les corporations ne sont pas représentées comme elles le souhaiteraient, surtout à Paris, où elles ne trouvent pas de place dans la représentation. À ce qui apparaît comme une injustice s'ajoute la contagion introduite par le nouvel état d'esprit, puisque une partie des maîtres se voit comme le tiers état soumis et résistant aux privilégiés, la formulation est évidemment reprise à leur compte par les compagnons, mais contre leurs patrons. Ce glissement de sens des mots amène les corporations à se proclamer du côté de la Révolution en marche, tout en faisant appel à une réglementation intelligente de la part de l'État. Corporatif et révolutionnaire tentent de se conjuguer, d'autant plus que les ébranlements de la nuit du 4 août et de la Déclaration des droits de l'homme posent la question de la survie des corporations. Mises à l'écart du débat dans un premier temps, alors que certains ouvriers estiment que dorénavant elles n'ont plus d'existence possible, elles subissent ce nouveau traumatisme de façon complexe. Les maitres dénoncent, évidemment, le chaos, mais ils se posent aussi en révolutionnaires, luttant eux aussi contre les " aristocrates » et se posent en garants de l'ordre social que les demandes ouvrières commencent à mettre à mal. La corporation est nuisible en tant qu'ordre intermédiaire, elle garde cependant une utilité en garantissant la qualité des ouvrages et en encadrant les compagnons. Sur ces deux points, les municipalités, dont celle de Paris, se retrouvent du côté des maîtres 
dès 1790 , et font face aux associations ouvrières contestatrices qui naissent à cette époque.

7 Dans cette difficile conciliation des principes révolutionnaires et de la vie quotidienne, la question des corporations devient une des pierres de touche des choix politiques, car le langage politique est interprété librement par toutes les parties, qui se réclament de leur nouveau statut de citoyen et ne veulent pas se retrouver assimilées à la ContreRévolution. Dans les luttes sociales de nouvelles qualifications sont usitées, si bien que les ouvriers sont dénoncés comme des «brigands», tandis que les maîtres deviennent des "monstres aristocratiques". Mais la crainte de la subversion ouvrière est primordiale devant la naissance de nouvelles formes associatives qui inquiètent maîtres, administrateurs et juges de paix. L'exemple est donné par l'Union fraternelle des charpentiers qui se constitue mi-amicale, mi-syndicat, et qui se retrouve, par force, dans la mouvance du courant cordelier. Les maîtres de leur côté créent les structures clés de la sans-culotterie. Dans ces échanges, les positions sont souvent contradictoires, les modérés sont plutôt libéraux économiquement, mais n'entendent pas céder aux pressions ouvrières; les courants plus extrémistes qui ont peur d'une dissolution du lien social par le pouvoir de l'argent, réclament l'interventionnisme de l'État soit pour protéger les ouvriers, soit pour empêcher les troubles. La question est tranchée par le décret Allarde qui, dans une optique essentiellement fiscale, supprime les corporations et crée la patente comme moyen d'accession au patronat, puis par la loi Le Chapelier, qui interdit toute association dans une optique répressive contre les menaces de subversions. S'agit-il comme le dit Kaplan d'une mesure d'inspiration révolutionnaire qui règle un problème d'Ancien Régime ou comme l'affirment des critiques royalistes d'une mesure imitant l'Ancien Régime et traitant un problème né de la Révolution? La question reste ouverte, sauf à dire que Le Chapelier n'est pas le visionnaire porteur des aspirations de la bourgeoisie industrielle du XIX ${ }^{e}$ siècle, mais bien le juriste, porté par un courant d'opinion ancien, qui établit le contrôle social réclamé par la saniorpars.

8 Les questions liées au travail ne sont plus ainsi traitées spécifiquement, mais dans un cadre politique, ce qui explique que patrons et ouvriers s'engagent dans l'univers politique, nommément dans la sans-culotterie, qui est l'objet d'un chapitre ultime dans lequel l'auteur propose d'y voir le lieu où les patrons exercent leur contrôle sous couvert d'unanimisme patriotique. L'histoire agitée des corporations dans la vie française est l'occasion de montrer comment les arguments et les positions peuvent s'échanger au fil des luttes sociales et politiques et trouver des résonances inattendues. Le livre se veut clairement de ce point de vue une leçon de méthode. À de nombreuses reprises, l'auteur s'engage dans des discussions historiographiques, le plus souvent menées dans l'épaisseur des notes mises en fin d'ouvrage, mais le dernier chapitre est pour l'essentiel consacré à la discussion des thèses de Soboul, Burstin, Sonenscher et Sewell sur la formation de la sans-culotterie. Ce qui est défendu ici est d'une part qu'il n'y a pas à chercher d'explications linéaires et simplistes, mais plutôt à comprendre des processus contradictoires qui débouchent sur des formations imprévues. La naissance de la sans-culotterie est pour l'auteur l'exemple de ce précipité chimique imprévisible qui, sous l'effet des nouvelles conditions provoquées par la Révolution, naît de la rencontre entre des attentes et des courants anciens, métamorphosés par l'événement. Il est possible de discuter dans le détail de la compréhension de la sans-culotterie, reste que la démarche est convaincante, liant étroitement structure et événement, ne se perdant pas dans des recherches généalogiques illusoires, dépassant ce qui est posé comme contradictions, insistant sur les mutations de sens des mots et des concepts, 
récusant les jugements trop rapides. Il rappelle notamment qu'il n'y a pas lieu de s'étonner qu'il y ait eu accord sur la loi Le Chapelier, dans la mesure où les données du débat étaient bien connues depuis des décennies, la loi étant de ce point de vue un aboutissement attendu. De la même façon, qu'il ait fallu attendre 1791 pour la voir promulguer n'est pas non plus un sujet d'étonnement, dans la mesure où les urgences étaient plus grandes dans d'autres domaines.

Deuxième leçon du livre, l'auteur attire à maintes occasions l'attention, sur la délicate articulation entre discours et "réalité » et la compréhension des représentations des acteurs de l'histoire. Il n'y a pas lieu de prendre au pied de la lettre les discours des corporations ou des ouvriers à aucun moment de l'histoire, il est même nécessaire d'introduire un "coefficient de dédramatisation " sur toutes les réclamations, pour tenir compte de la complexité du réel, de la désunion de chaque camp, de la part d'invention qui se trouve dans ces discours. La discussion sur la nature de la sansculotterie est au cœur de cette réflexion, puisque l'auteur conteste les analyses qui prennent sans précaution les proclamations sans tenir compte suffisamment des structures sociales sous-jacentes cachées précisément par ces allégations. Il est possible de le suivre dans cette voie qui ne prend pas au pied de la lettre les dénonciations de l'absolutisme royal, puisque l'on voit les agents du roi engagés dans des négociations avec leurs partenaires, mais à l'intérieur même de l'administration, comme il ne faut pas croire les déclarations des tenants des corporations, d'autant plus fortes qu'elles sont données au moment d'une refondation qui doit faire oublier les tares antérieures. Plus profondément encore, l'analyse est stimulante dans ce qui est dit de la nature véritable du lien corporatif, dont l'efficacité est moins économique que sociale. Le discours tenu se situait bien au niveau économique, mais la cohésion cherchée était idéologique plus qu'économique, car de tous temps les écarts à la règle étaient considérables et bien connus. En revanche, l'atteinte aux normes admises entraînaient ipso facto un renversement des valeurs, qui allait toucher toute l'épaisseur de la société au-delà des seules structures économiques corporatives. C'est en cela que l'épisode traumatique de 1786 est créateur d'une nouvelle donne sociale et politique, engageant le pays dans une désacralisation et une décorporéification de la société, annonçant l'atomisation des liens sociaux provoqués par la Révolution. Là encore, le livre apporte des éléments de réflexion en montrant bien comment ces idées qui travaillent profondément le tissu social ne sont pas véritablement opératoires avant que les événements factuels dés 14 juillet et 4 août 1789 n'aient clairement changé les choses. Comme dans une réaction chimique, les éléments dispersés se précipitent et créent de nouvelles formes. De ce point de vue, l'auteur donne certes raison à ceux qui voient le début de la Révolution bien avant 1789, la « matrice de la Révolution » est présente dès 1786, mais il renforce surtout ceux qui insistent sur la cristallisation événementielle, indispensable à la mutation des esprits. De ruptures en cristallisation, de malentendus en fixations idéologiques inédites, le livre explore ainsi les ruses de la raison économique et politique qui ont traversé les tactiques des maîtres, des compagnons, des administrateurs et des parlementaires, se situant bien au-delà du champ apparemment limité d'une étude du monde du travail ou d'une réflexion sur la « fin des corporations ", comme le titre pourrait le laisser croire. C'est tout le processus de la Révolution française qui est ainsi éclairé au travers d'un domaine particulier, ce qui fait un des intérêts essentiels du livre.

10 Il convient enfin d'insister sur l'aspect peut-être le plus paradoxal de l'ouvrage, et qu'il ne faudra pas oublier dans les discussions à venir. D'un bout à l'autre, l'auteur insiste 
sur l'aspect heuristique de sa démarche, sur sa volonté de proposer une interprétation, et il rappelle avec une insistance parfois inquiétante pour le lecteur attendant des conclusions définitives que, malgré les notes de référence qui donnent lieu à une centaine de pages, il ne s'appuie que sur des dépouillements limités, sur des «miettes d'archives ", qu'il faudra donc multiplier les études locales et les analyses fines. Ce qui est en jeu ici n'est pas la description achevée d'un processus, mais bien l'ébauche d'un cadre interprétatif qui reste à démontrer dans les détails. À n'en pas douter, ce livre va relancer de nombreuses discussions tant sur les méthodes de l'histoire sociale, que sur les conditions mêmes de la naissance de la sans-culotterie, il participe en cela au renouvellement et à l'actualisation des problématiques de recherche et mérite d'être lu attentivement. 